\title{
A New Fractal Hyperspectral Image Compression Algorithm
}

\author{
Yun Chen, Ruidong Gao \\ School of Instrumentation Science and Optoelectronics Engineering, Beihang University, Beijing 100191, China \\ chenyun1121@163.com
}

\begin{abstract}
Because of the great data of the hyperspectral image, it is not good for storage and transmission. To work out a high efficiency compression method for hyperspectral image is essential. Prediction encoding is easy to realize and has been studied widely in the hyperspectral image compression field. High compression ratio, resolution independence and fast decoding speed are the main advantages of fractal coding which makes full use of the local self-similarity existing in images and is considered as a promising compression method. However, the application of fractal coding in the hyperspectral image compression field is not widespread. In this paper, we propose a new fractal hyperspectral image compression algorithm. Considering the noises which exist in the hyperspectral image and the integrity of decompression, Firstly, intra-band prediction is implemented to the first band. Checking the noise whether the current encoding band is greater than the threshold value. If it is greater, the current encoding band will be considered to be a noise band and perform intra-band prediction. The first non-noise band which precisely followed the noise band performs the intraband prediction encoding. The rest of the bands will be encoded by modified fractal coding algorithm. The proposed algorithm can effectively exploit the temporal-spatial correlation in hyperspectral image, since each range block is approximated by the domain block which is of the same size as the range block in the adjacent band. Experimental results indicate that the proposed algorithm provides very promising performance at low bitrate.
\end{abstract}

Keywords-Hyperspectral image; Fractal; Prediction coding.

\section{INTRODUCTION}

Hyperspectral remote sensing has been widely applied in many areas such as geology, ecology, atmospheric study, and soil study. It is playing an increasingly important role [1]. Currently, prediction-based, transformation-based and vector quantization-based are the main hyperspectral image compression methods. Reference [2] applies median prediction algorithm for intra prediction, and linear prediction and context prediction mixed algorithm for inter prediction to achieve lossless compression of hyperspectral images. Reference [3] which applies an improved KLT in decorrelation of hyperspectral images doesn't achieve a significant impact on reconstructed image quality. Shen-En Qian [4] which does not require full search and greatly reduces the complexity provides a fast vector quantization algorithm to improve the generation efficiency of codebook.

Since fractal compression was proposed, there have been a lot of applications. It was not only applied in image compression [5][6], but also was widely applied in video compression recently. Reference [7] explored the fractal video sequences coding in the context of region-based. Reference [8][9] introduced the fractal into the multi-view video compression.

As we observe that there exist strong similarities between adjacent bands of hyperspectral images, fractal coding will be suitable for hyperspectral image compression. In this paper, we combine prediction and fractal coding for hyperspectral image compression.

The rest of the paper is organized as follows. Section II provides the basis of fractal image coding. Section III is the proposed hybrid prediction and fractal compression algorithm. The experimental results are presented in Section IV. And finally the conclusions are outlined in Section V.

\section{FRACTAL IMAGE CODING BASIS}

\section{A. Background of Fractal Compression}

Jacquin described the first practical fractal image compression algorithm based on a Partitioned Iterated Function System (PIFS) [10] [11] and divided the image I into two sizes of blocks [12]. The current frame is made up of range blocks $\left(R_{m(i)}\right)$ each of which is consist of $\mathrm{B} \times \mathrm{B}$ pixels and allows non-overlapping and covering the entire image. The decode frame is made up of the $2 \mathrm{~B} \times 2 \mathrm{~B}$ pixels which are called domain block $\left(D_{m(i)}\right)$ and allow overlapping. All the domain blocks form a domain pool. The image can be represented by

$$
I=\left\{R_{m(i)}\right\}_{0 \leq i \leq N}
$$

Where $N$ is the total number of range blocks.

Each range block $R_{m(i)}$ shall be approximated by a certain domain block $D_{m(i)}$ with the transformation $\omega_{i}: D_{m(i)} \rightarrow R_{m(i)}$, which is composed of spatial contraction transformation $\gamma_{i}$ and grayscale modification transformation $\lambda_{i}$.

$$
\omega_{i}=\lambda_{i} \times \gamma_{i}
$$

$\omega_{i}$ is often a contracted affine transformation, its general form is: 


$$
\omega_{i}\left(D_{m(i)}\right)=\lambda_{i}\left(\gamma_{i}\left(D_{m(i)}\right)\right)=s_{i} \cdot t_{k}\left(\gamma_{i}\left(D_{m(i)}\right)\right)+o_{i}
$$

$\gamma_{i}$ uses the average value of four adjacent pixels to get B $\times \mathrm{B}$ pixel block $\hat{D}_{m(i)}$. As shown in Fig. 1, the average value of four adjacent pixels is:

$$
\hat{d}_{h, v}=\frac{1}{4}\left(d_{2 h, 2 v}+d_{2 h+1,2 v}+d_{2 h, 2 v+1}+d_{2 h+1,2 v+1}\right)
$$

Where $d_{h, v}$ and $\hat{d}_{h, v}$ represent pixel values of $D_{m(i)}$ and $\hat{D}_{m(i)}$ at the pixel position $(h, v)$, respectively.

$\lambda_{i}$ introduces the grayscale adjustment parameter $S_{i}$, grayscale offset parameter $O_{i}$, and isometric transformation $t_{k}$ in order to get a better approximation of $R_{m(i)}$ by further modifying the grayscale of $D_{m(i)}$. The general form of $\lambda_{i}$ is:

$$
\lambda_{i}\left[\begin{array}{c}
x \\
y \\
z(x, y)
\end{array}\right]=\left[\begin{array}{lll}
a & b & 0 \\
c & d & 0 \\
0 & 0 & s_{i}
\end{array}\right]\left[\begin{array}{c}
x \\
y \\
z(x, y)
\end{array}\right]+\left[\begin{array}{c}
0 \\
0 \\
o_{i}
\end{array}\right]
$$

Where $a, b, c$ and $d$ determine 8 kinds of pixel rearrangements ( 4 kinds of rotation and 4 kinds of flip); $S_{i}$ and $o_{i}$ represent brightness adjustment and brightness offset respectively.

The fractal coding is aimed to look for the best matching block $D_{m(i)}$ and block transformation $\omega_{i}$ for each, so that $R_{m(i)}^{\prime}=\omega_{i}\left(D_{m(i)}\right)$ and $R_{m(i)}$ are as close as possible in the given distortion metric, namely

$$
d\left(R_{m(i)}, \omega_{i}\left(D_{m(i)}\right)\right)=\min \left\{d\left(R_{m(i)}, \omega\left(D_{m(i)}\right)\right)\right\}
$$

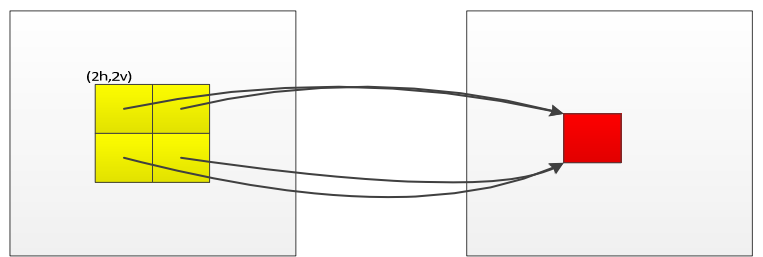

Domain blocks

Range blocks

Figure 1. The mapping from domain to range blocks.

\section{III.HYBRID PREDICTION AND FRACTAL COMPRESSION}

In order to take full advantages of spatial and spectral correlations of hyperspectral image, we propose a new fractal hyperspectral image compression algorithm.

Because there are a part of noises in the hyperspectral image and in order to ensure the accuracy of the data at the same time, it is necessary for the noise image to be special processed. Before we encode a band, we need to judge whether it is the first band. If it is, intra-band prediction coding will be used. If not, we need to judge whether the noise is greater than the threshold value. If it is, the current band will be coded by intra-band prediction. If not, we need to judge whether the noise of previous band is greater than the threshold value. If it is, we use intra-band prediction to code the current band. Otherwise, inter-band fractal coding will be used and then go on encoding the next band. Then the prediction errors and fractal residuals are further transformed, quantified and entropy encoded, and the fractal parameters are also entropy encoded to improve the compression efficiency. The compression scheme divides each band into range blocks with the size of $16 \times 16$. The block diagram for this scheme is shown in Fig. 2.

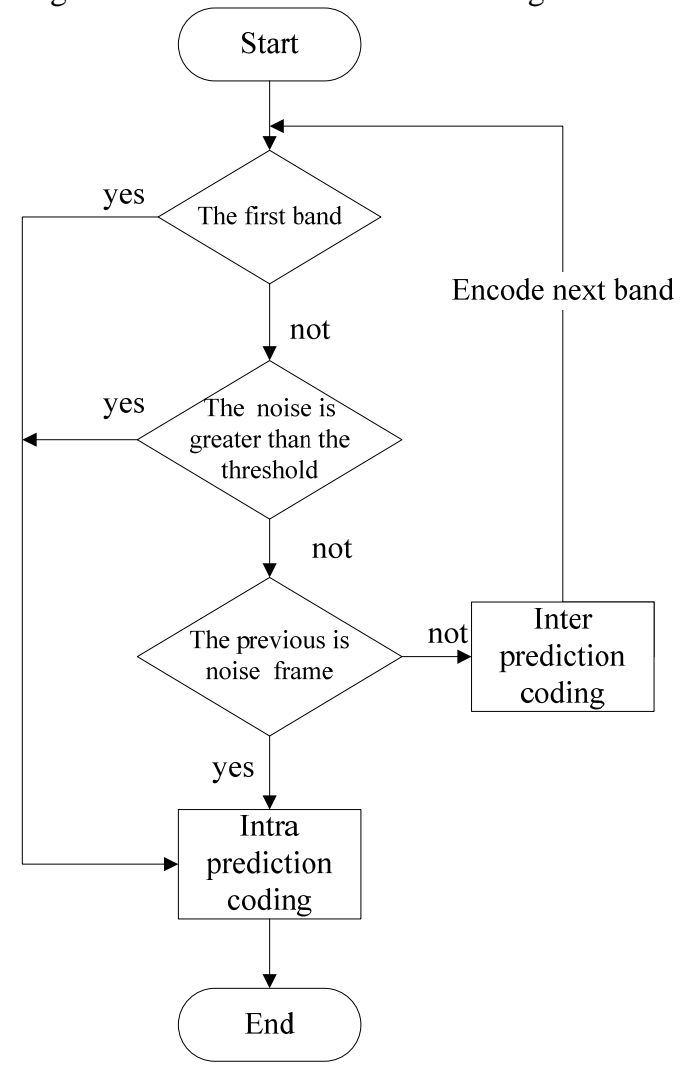

Figure 2. Block diagram of the proposed scheme

\section{A. Intra-Band Prediction}

In this section, we describe the intra-band prediction design of our scheme. The main purpose is to remove spatial correlation as well as obtaining a high quality decoded reference band. 
In view of the H.264/AVC frame prediction coding performance in removing spatial correlation, the noise band and the first non-noise band which precisely follows the noise band apply the intra-band prediction coding. Intraband prediction makes full use of spatial correlation of adjacent blocks and the reconstruction of left and up pixels of the current block. The intra-band prediction is divided into two modes, $16 \times 16$ and $4 \times 4$.

$16 \times 16$ MODE: For the band which has relatively flat texture, it is divided into $16 \times 16$ pixels. There are four candidate prediction modes: Mode0 (Vertical prediction), Mode1 (horizontal prediction), Mode2 (DC prediction), Mode3(plane prediction). As shown in Fig. 3.

$4 \times 4$ MODE: For the band which has complicated flat texture, it is divided into $4 \times 4$ pixels. There are nine candidate prediction modes: Mode0(Vertical), Mode1(horizontal), Mode2(DC), Mode3(diagonal downleft), Mode4(diagonal down-right), Mode5(vertical-right), Mode6(horizontal-down), Mode8(horizontal-up). As shown in Fig. 4.
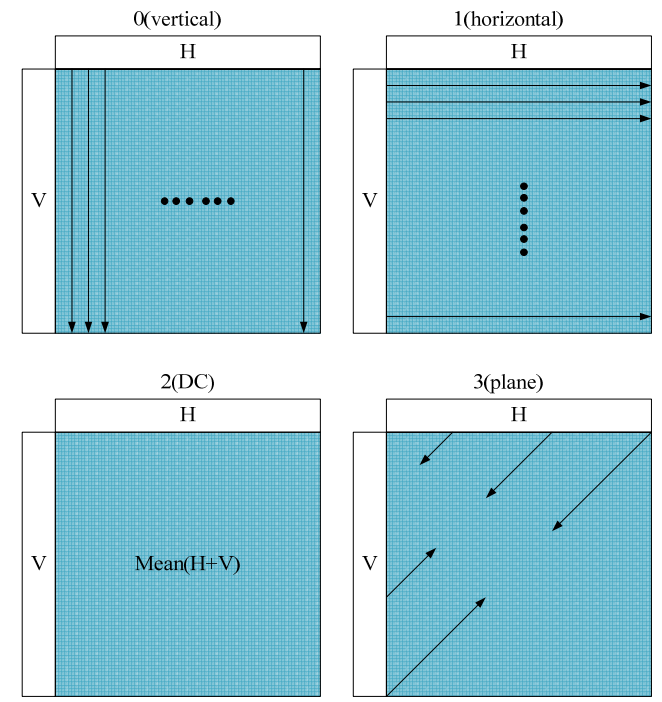

Figure 3. Prediction mode of $16 \times 16 \mathrm{MODE}$

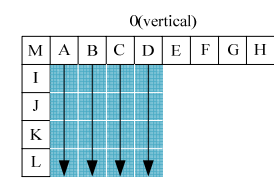

3 (diagonal down-left)

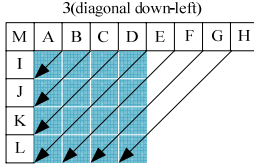

6(horizontal-down)
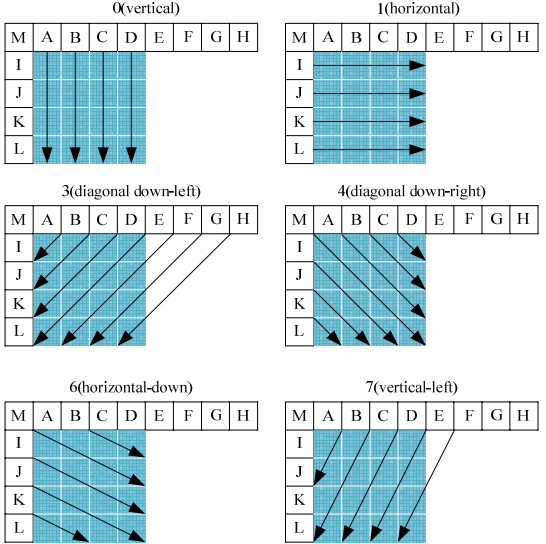

4(diagonal down-right)

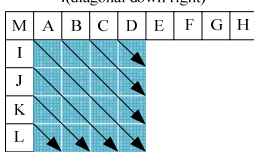

7 (vertical-left)
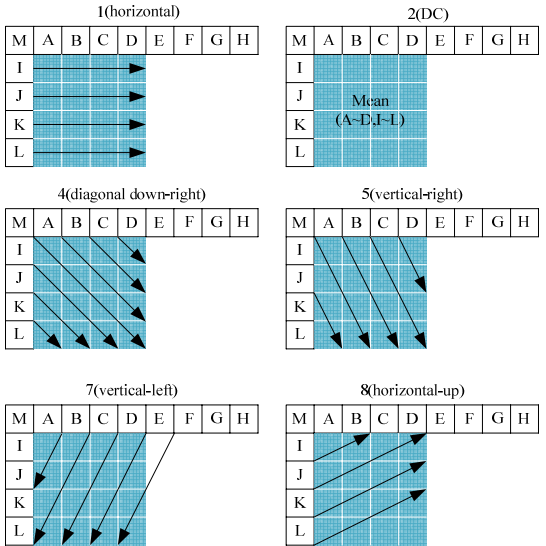

5 (vertical-right)

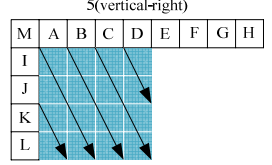

8(horizontal-up)

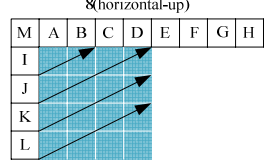

Figure 4. Prediction mode of $4 \times 4 \mathrm{MODE}$

The prediction mode with the minimum sum of squared difference (SSD) is chosen as the final prediction mode. The SSD is calculated as follows.

$$
\operatorname{SSD}(\mathrm{s}, \mathrm{c})=\sum_{\mathrm{x}=1}^{4} \sum_{\mathrm{y}=1}^{4}(\mathrm{~s}(x, y)-\mathrm{c}(\mathrm{x}, \mathrm{y}))^{2}
$$

Where, $\mathrm{s}(\mathrm{x}, \mathrm{y})$ represents the source signal at position ( $\mathrm{x}$, $\mathrm{y}) ; \mathrm{c}(\mathrm{x}, \mathrm{y})$ represents the reconstruction signal at position ( $\mathrm{x}$, y) using corresponding prediction mode.

\section{B. Inter-band Fractal Coding}

In order to remove the existing spectral redundancy in hyperspectral image, we have made some modifications to the basic block-based fractal image compression scheme. In our scheme, each range block is as large as the domain block in the adjacent band. Considering the adjacent bands have similar spatial topology structures, the isometric transformation process can be omitted. Statistics also shows that the best matching $\mathrm{D}$ block appears near $\mathrm{R}$ block with a large probability [13], so it's not necessary to use full search. In our scheme, the search range of block matching is reduced to a small region centering the corresponding position of $\mathrm{R}$ block, as shown in Fig. 5. 


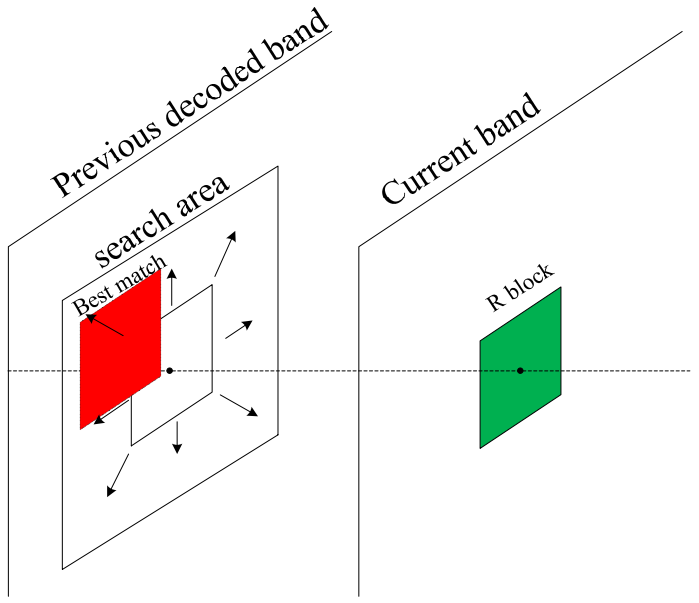

Figure 5. Searching the best block.

The specific steps for inter-band fractal coding are as follows:

Step I: The current hyperspectral band image is divided into non-overlapping $\mathrm{R}$ blocks with the size of $16 \times 16$, and all $\mathrm{R}$ blocks can cover the whole band image.

Step II: Encode each R block in turn.

Searching the best match for the block in the search area of the adjacent band is the most important work. We need to compute the MSE of different size, such as $16 \times 16,16 \times 8$, $8 \times 16,8 \times 8,8 \times 4,4 \times 8,4 \times 4$. The size which has the minimum MSE will be the best choice.

$$
\operatorname{MSE}(s, o)=\frac{1}{N} \sum_{i=1}^{N}\left[r_{i}-\left(s \cdot d_{i}+o\right)\right]^{2}
$$

Where $r_{i}$ represents the pixel value of the current $\mathrm{R}$ block, $d_{i}$ represents the pixel value of the corresponding matching D block, $N$ represents the number of pixels of the current R block, $S$ is the scale factor, and $O$ is the offset factor.

$S$ and $O$ are defined as follows:

$$
\left\{\begin{array}{l}
S=\frac{N\left(\sum_{i=1}^{N} r_{i} \cdot d_{i}\right)-\sum_{i=1}^{N} r_{i} \sum_{i=1}^{N} d_{i}}{N \sum_{i=1}^{N} d_{i}^{2}-\left(\sum_{i=1}^{N} d_{i}\right)^{2}} \\
o=\frac{1}{N}\left(\sum_{i=1}^{N} r_{i}-s \sum_{i=1}^{N} d_{i}\right)
\end{array}\right.
$$

Step III: Repeat step II until all the R blocks of the current hyperspectral band have found the best matching blocks.

\section{IV.EXPERIMENTAL RESULT}

Two AVIRIS hyperspectral data cubes, "Cuprite" and "Low Altitude", derived from JPL are used. The size of the data cubes was originally 512 lines $\times 614$ pixels with 224 spectral bands and 1087 lines $\times 614$ pixels with 224 spectral bands respectively. In this paper, a subset of them with 512 lines $\times 512$ pixels with all 224 bands was tested. PSNR is used to measure the quality of the compression data. It is defined as follows:

$$
P S N R=10 \log _{10} \frac{255^{2}}{M S E}
$$

MSE is calculated as:

$$
M S E=\left[\frac{1}{N_{x} N_{y} N_{z}} \sum_{x=1}^{N_{x}} \sum_{y=1}^{N_{y}} \sum_{z=1}^{N_{z}}\left[f^{\prime}(x, y, z)-f(x, y, z)\right]^{2}\right]^{1 / 2}
$$

$f(x, y, z)$ and $f^{\prime}(x, y, z)$ are respectively the pixel values of the original and the reconstructed data of band $\mathrm{z}$ at location (x,y), $N_{y}$ is the total number of lines in the data cube, $N_{x}$ is the total number of pixels per line, $N_{z}$ is the total number of bands.

Fig. 6(a) shows the 10th band of "Cuprite" truncated from the original data cube for the experiment, and Fig. 6(b) shows the decoded result image at $0.1 \mathrm{bpppb}$. As seen in Fig. 6 , there is almost no difference of subjective quality between the compressed image and the original image. 


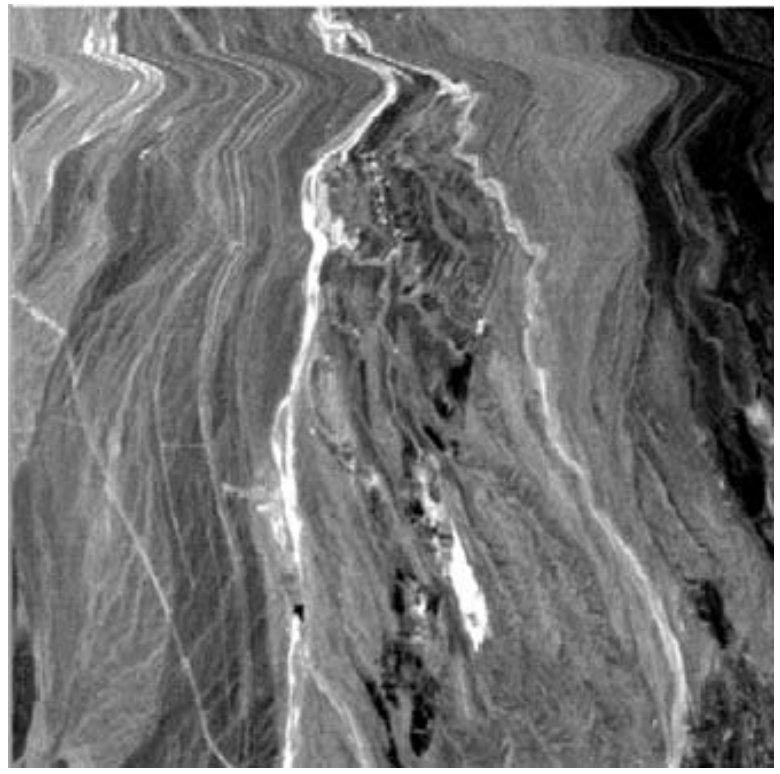

(a) Original image

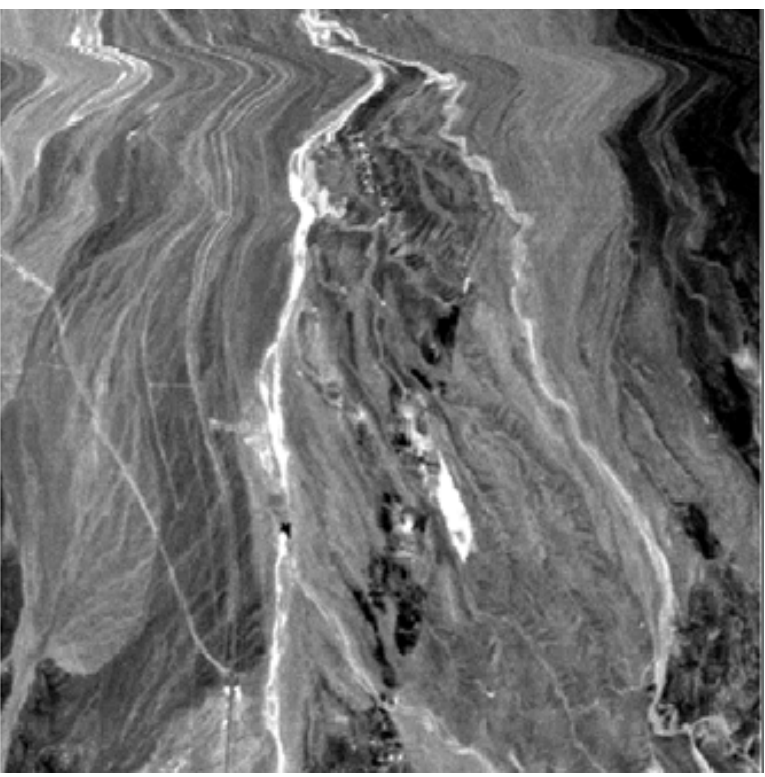

(b) Decoded image

Figure 6. The 115th band image of "Cuprite"

TABLE I. AVERAGE PSNR COMPARISON FOR “LOW AltitUdE” (UNIT: dB)

\begin{tabular}{cllll}
\hline Code rate (bpp) & 0.1 & 0.15 & 0.2 & 0.25 \\
AT-3DSPIHT [14] & 47.5 & 49.5 & 51.5 & 52.61 \\
Proposed & $\mathbf{4 6 . 6 8}$ & $\mathbf{5 2 . 0 1}$ & $\mathbf{5 4 . 1 1}$ & $\mathbf{5 5 . 2 3}$ \\
\hline
\end{tabular}

TABLE II. AVERAGE PSNR COMPARISON FOR “CUPRITE” (UNIT: dB)

\begin{tabular}{cllrr}
\hline Code rate (bpp) & 0.05 & 0.1 & 0.15 & 0.2 \\
AT-3DSPIHT [14] & 44.25 & 45.47 & 46.72 & 48.05 \\
Proposed & $\mathbf{4 5 . 5 7}$ & $\mathbf{4 8 . 3 5}$ & $\mathbf{5 0 . 8 7}$ & $\mathbf{5 2 . 5 4}$ \\
\hline
\end{tabular}

The PSNR comparison results at different bitrates are as shown in Table I and Table II. Experimental results show that the PSNR of "Low Altitude" compressed by the proposed algorithm is slightly lower than that of AT3DSPIHT at $0.1 \mathrm{bpp}$. Furthermore, the values of PSNR are all higher than the contrast algorithm at other code rates. Compared with AT-3DSPIHT algorithm, the PSNR of the proposed algorithm is increased by $1.73 \mathrm{~dB}$ in average.

The PSNR of "CUPRITE" compressed by the proposed algorithm are all higher than the contrast algorithm. Compared with AT-3DSPIHT algorithm, the PSNR of the proposed algorithm is increased by $3.22 \mathrm{~dB}$ in average.

The encoding time is used to measure the encoding complexity of the algorithm. The time of the proposed scheme is just about $20 \%$ of the reference [15]. So the proposed scheme greatly reduces the encoding time.

\section{CONCLUSION}

From the experimental results, conclusion can be made that the algorithm proposed achieves effective compression of hyperspectral image at low bit rate, the decoded quality is

greatly improved. Besides, the encoding complexity also has reduced. The proposed hybrid prediction and fractal hyperspectral image compression algorithm is a breakthrough to the traditional algorithm framework of hyperspectral image compression, providing a commendable solution for lossy compression of hyperspectral image.

\section{ACKNOWLEDGMENT}

This project is funded by the National Natural Science Foundation of China (NSFC) under grants No. 61375025.

\section{REFERENCES}

[1] N. R. M. Noor, T. Vladimirova. Investigation into lossless hyperspectral image compression for satellite remote sensing. International Journal of Remote Sensing, vol. 34, no. 14, pp. 50725104, 2013.

[2] Y. Liang, J. Li, K. Guo. Lossless compression of hyperspectral images using hybrid context prediction. Optics Express, vol. 20, no. 7, pp. 8199-8206, 2012.

[3] B. Penna, T. Tillo, E. Magli, G. Olmo. Transform coding techniques for lossy hyperspectral data compression. IEEE Transactions on Geoscience and Remote Sensing, vol. 45, no. 5, pp. 1408-1421, 2007. 
[4] S. -E. Qian. Hyperspectral data compression using a fast vector quantization algorithm. IEEE Transactions on Geoscience and Remote Sensing, vol. 42, no. 8, pp. 1791-1798, 2004.

[5] S. P. Zhu, Y. Gao. Noncontact 3-D coordinates measurement of cross-cutting feature points on the surface of a large-scale workpiece based on the machine vision method. IEEE Transactions on Instrumentation and Measurement, vol. 59, no. 7, pp. 1874-1887, July 2010.

[6] S. P. Zhu, J. C. Fang, R. Zhou, J. H. Zhao, W. B. Yu. A new noncontact flatness measuring system of large 2-D flat workpiece. IEEE Transactions on Instrumentation and Measurement, vol. 57, no. 12, pp. 2891-2904, December 2008.

[7] S. P. Zhu, Y. S. Hou, Z. K. Wang, K. Belloulata. Fractal video sequences coding with region-based functionality. Applied Mathematical Modelling, vol. 36, no. 11, pp. 5633-5641, 2012.

[8] S. P. Zhu, L. Y. Li, J. Q. Chen, K. Belloulata. An efficient fractal video sequences codec with multiviews. Mathematical Problems in Engineering, vol. 2013, Article number 853283, pp. 1-8, 2013.

[9] S. P. Zhu, D. Y. Zhao, L. Zhang. A novel high efficiency fractal multiview video codec. Mathematical Problems in Engineering, vol. 2015, Article number 613714, pp. 1-12, 2015.
[10] M. F. Barnsley. Fractals Everywhere. Academic Press, New York, 1988.

[11] M. F. Barnsley, S. Demko. Iterated function systems and the global construction of fractals. Proceedings of the Royal Society of London A Mathematical and Physical Sciences, A399, pp. 243-275, 1985.

[12] A. E. Jacquin. Image coding based on a fractal theory of iterated contractive image transformations. IEEE Transactions on Image Processing, vol. 1, no. 1, pp. 18-30, 1992.

[13] A. C. Miguel, R. E. Ladner, E. A. Riskiny. Predictive coding of hyperspectral images. Springer US, 2006.

[14] X. Tang, C. Sungdae, W. A. Pearlman. 3D set partitioning coding methods in hyperspectral image compression. Proceedings of the IEEE International Conference on Image Processing (ICIP), Barcelona, Spain, vol. II, pp. 239-242, 2003.

[15] W. Pan, Z. Yi, A. Lu. A compression algorithm of hyperspectral remote sensing image based on 3-D wavelet transform and fractal. Proceedings of 3rd International Conference on Intelligent System and Knowledge Engineering, vol. 1, pp. 1237-1241, 2008. 